\title{
Optimal Power System and Grid Interface Design Considerations for the CLICs Klystron Modulators
}

\author{
Marija Jankovic ${ }^{1}$, Alan Watson ${ }^{1}$, Jon Clare ${ }^{1}$, Pat Wheeler ${ }^{1}$, and Davide Aguglia ${ }^{2}$ \\ ${ }^{1}$ University of Nottingham, Nottingham, UK, ${ }^{2} \mathrm{CERN}$, Geneva, Switzerland
}

Keywords: CLIC, grid interface, Power system

\begin{abstract}
The Compact Linear Collider (CLIC) is an electron-positron collider under study at CERN with the aim to explore the next generation of high precision/high energy particles physics. The CLIC's drive beams will be accelerated by approximately 1300 klystrons, requiring highly efficient and controllable solid state capacitor discharge modulators. Capacitor charger specifications include the requirement to mask the pulsed effect of the load from the utility grid, ensure maximum power quality, control the derived DC voltage precisely (to maximize accuracy for the modulators being implemented), and achieve high efficiency and operability of the overall power system.

This paper presents the work carried out on the power system interface for the CLIC facility. In particular it discusses the challenges on the utility interface and analysis of the grid interface converters with regards to required functionality, efficiency, and control methodologies.
\end{abstract}

Presented at: IEEE IPMHVC, 1-5 June 2014, Santa Fe, US

Geneva, Switzerland

January, 2015 


\title{
Optimal Power System and Grid Interface Design Considerations for the CLICs Klystron Modulators
}

\author{
Marija Jankovic $^{1}$, Alan Watson ${ }^{1}$, Jon Clare ${ }^{1}$, Pat Wheeler ${ }^{1}$, and Davide Aguglia ${ }^{2}$ \\ ${ }^{1}$ University of Nottingham, Nottingham, NG7 2RD, UK \\ ${ }^{2}$ CERN - European Organization for Nuclear Research, Geneva, CH-1211, Switzerland
}

\begin{abstract}
The Compact Linear Collider (CLIC) is an electronpositron collider under study at CERN with the aim to explore the next generation of high precision/high energy particles physics. The CLIC's drive beams will be accelerated by approximately 1300 klystrons, requiring highly efficient and controllable solid state capacitor discharge modulators. Capacitor charger specifications include the requirement to mask the pulsed effect of the load from the utility grid, ensure maximum power quality, control the derived DC voltage precisely (to maximize accuracy for the modulators being implemented), and achieve high efficiency and operability of the overall power system.

This paper presents the work carried out on the power system interface for the CLIC facility. In particular it discusses the challenges on the utility interface and analysis of the grid interface converters with regards to required functionality, efficiency, and control methodologies.

Index Terms — CLIC, grid interface, Power system
\end{abstract}

\section{INTRODUCTION}

The Compact Linear Collider is the highest energy linear electron-positron collider under study at CERN with the aim to explore the next generation of high precision/high energy particles physics [1]. The CLIC is based on a two beam principle, where drive beams are accelerated with around 1300 klystrons and the main beams are accelerated by deceleration energy from the drive beams. In the CLIC, the acceleration power is pulsed, feeding the klystrons with the high voltage pulses $(150-180 \mathrm{kV})$ with $140 \mu \mathrm{s}$ duration and $50 \mathrm{~Hz}$ repetition rate. The peak power per klystron is approximately $29 \mathrm{MW}$ resulting in an overall peak power of $38 \mathrm{GW}$. The grid interface of the drive beam klystron modulators should ensure that a constant power of $\sim 280 \mathrm{MW}$ is drawn from the grid. The specifications of the grid interface and pulse forming system for the CLIC's klystrons is given in [2].

The efficiency of the power system as a grid interface should be very high (above $96 \%$ ) due to high power demand and limited available power from the local grid (connection is at transmission voltage level). The power system should also provide high availability for the accelerator. Availability is achieved by over dimensioning, adding redundancy and using topologies with low mean time to repair (MTTR) and failure rates.

Power system design includes global integration, selection of the number of converters, transformers and other power system components, and the most suitable AC and DC voltage levels for the distribution. Design rules are set to achieve high efficiency, availability and reconfigurability of the network in case of a fault. CERN's technical and general integration constraints are taken into account. Power system design compromises and power converter challenges for the CLIC interface are presented in [3].

Power electronics design rules must respect the high efficiency and redundancy requirements. Supporting the dynamics of the pulsed capacitor discharge and low AC power fluctuation are the main objectives. Modular multi-level topologies are foreseen as the most suitable utility interface power converters for this application since they offer high efficiency, reliability and redundancy $[4,5]$.

The final solution is a result of technical-economic analysis and a compromise of the performance and price parameters.

\section{SYSTEM OPTIMISATION}

In order to respect the challenges associated with the grid and the modulators together with the performance parameters of the system, a global optimization approach must be undertaken. The candidate solutions for the power system grid interface, including both converter topologies and distribution equipment, are presented in [6]. Considering all potential structures presented in [6], and the required functionalities to be achieved, the optimal solution will be selected. Dedicated optimal grid layout is defined by the power system design approach, distribution voltage levels and quantity and ratings of the transformers, and converters, etc.

Optimization parameters driving the design are the overall efficiency, cost and integration issues and the reliability and availability of the accelerator. Fault currents in the system should not exceed the capabilities of standard distribution equipment. Converters topology selection and control is strongly coupled with the power system design. 
The optimization methodology is described with the graph in Figure 1. Grid specifications include the voltage level and power quality constraints such as maximum power fluctuation, the power factor and current harmonic content and maximum fault power. Klystron modulator specifications are defined by other collaboration teams working on pulse forming systems [7], and klystron design. In general, specifications relate to the DC voltage level, and pulse-to pulse voltage repeatability.

Based on the specifications, topological selections relating to the grid layout, voltage ratings, and number of power system components are presented. Some fundamental design rules are set, to support CERN's technical integration constraints and improving efficiency and redundancy. The converter topology is selected according to the required voltage and power ratings.

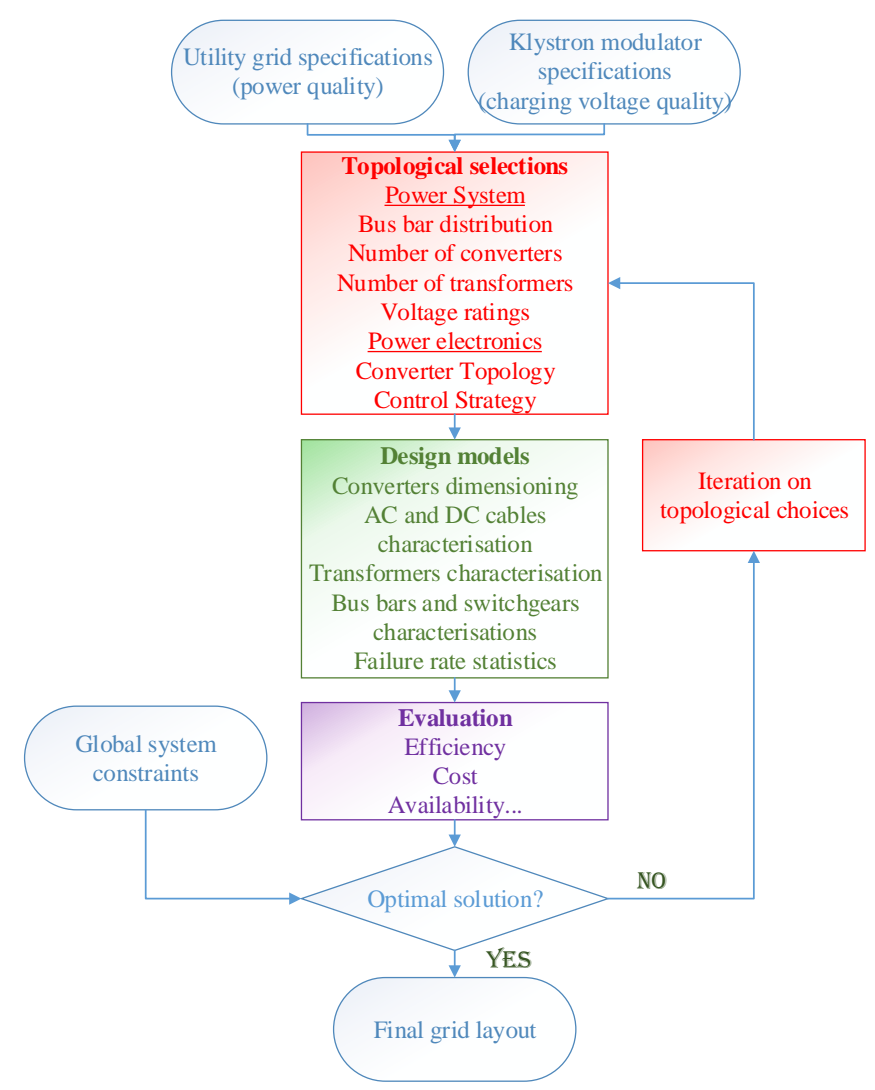

Figure 1. The optimization methodology diagram.

The next step is the derivation of the power system components, including efficiency, size, cost, and failure rate estimation. Evaluation of the overall power system performance is calculated taking into account efficiencies, impedances, failure rates, etc. of power system components. In the case that the global performance matches the system requirements, the optimal solution is found. Otherwise, the next iteration of the topological selections should be evaluated.

\section{POWER SYSTEM DESIGN}

The power system must be designed considering the grid parameters, which are presented in Table 1 . The DC voltage required for pulse forming structures should be in the range of $3.3 \mathrm{kV}$ to $20 \mathrm{kV}$. Thus the power system should include step down transformers and AC/DC converters. Pulse forming systems are distributed along the $2-2.5 \mathrm{~km}$ long drive beams, leaving the space for different spatial distribution approaches.

The candidate solutions presented in [6] are divided into the two main categories - single and double stage conversion. The main difference is that double stage conversion contains both AC/DC converters and step-down DC/DC converters. The expected efficiency of the DC/DC converters is lower than the efficiency of the medium voltage (MV) step down transformers, especially in cases of isolated converters that contain a transformer. Thus, only the single-stage conversion solutions are considered.

Table 1. Parameters of the French grid power provider.

\begin{tabular}{|c|c|}
\hline Grid voltage & $400 \mathrm{kV}$ \\
\hline Available power & $400 \mathrm{MVA}$ \\
\hline Maximum short circuit power & $14000 \mathrm{MVA}$ \\
\hline Resistance/reactance ratio & 0.07 \\
\hline Zero seq. impedance ratio & 0.822 \\
\hline Power fluctuation & $<2 \%$ \\
\hline Source distance & $\sim 500 \mathrm{~m}$ \\
\hline
\end{tabular}

A power system layout example is illustrated in the Figure 2. The $400 \mathrm{kV}$ grid voltage is connected to the outdoor substation via overhead lines. A certain number of HV/MV transformers are used to step down the voltage level. The HV/MV transformers are oil insulated, and typical power ratings are between 50 and $150 \mathrm{MVA}$. The number of outdoor HV/MV substations can be higher than one and distributed along the drive beams. At all voltage levels radial bus bar based distribution is applied. One of the design rules imposes that the highest possible voltage should be used for the distribution. Distributed HV/MV substations (400 kV distribution voltage) and distributed MV bus bars (MV distribution) along the drive beams support this design rule.

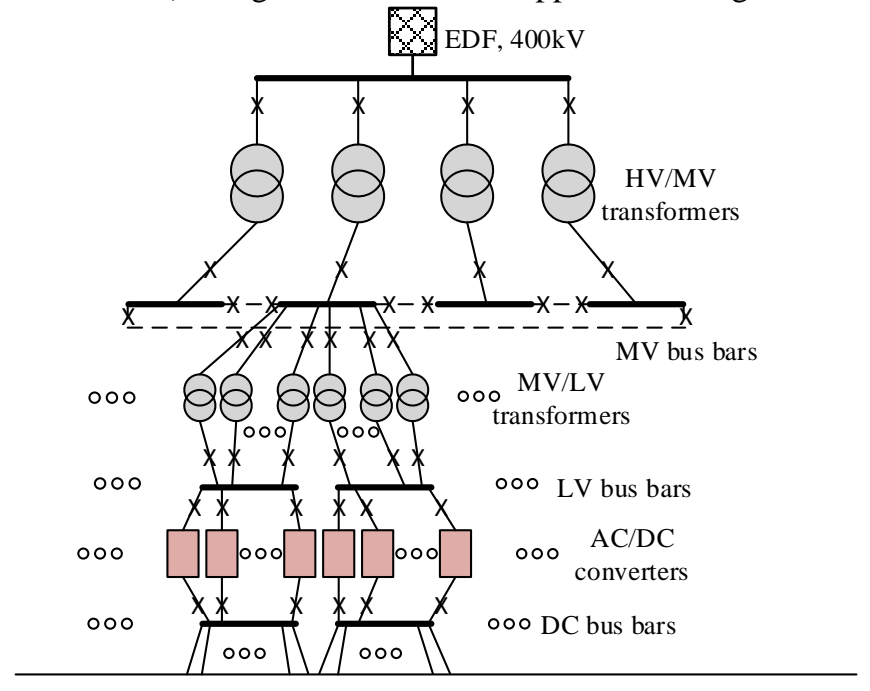

Klystron modulators

Figure 2. An example of the power system layout in case of single radially distributed bus bars.

The MV/LV transformers are usually considered to be "dry" 
typically using cast resin insulator, which is inexpensive when compared to oil dielectrics. Typical power ratings are between 2 and 4 MVA. Considering typical MV cables and protection ratings, the possible primary voltage ratings are $30 \mathrm{kV}(36 \mathrm{kV}$ equipment) and $20 \mathrm{kV}$ ( $24 \mathrm{kV}$ equipment). Depending on the required DC voltage the secondary transformer voltage is selected. In the case of $10 \mathrm{kV} \mathrm{DC}$ voltage, the AC voltage should be approximately $6 \mathrm{kV}$. Based on converter design, IGBT ratings, and cabling ratings, the considered DC currents are between $200 \mathrm{~A}$ and $1 \mathrm{kA}$.

All bus bars associated to the voltage levels corresponding to MV range and below are realized as enclosed switchgears, placed in indoor substations. Redundancy is achieved by parallel connection of transformers (or converters) outputs to a common bus bar. In the case when $n$ parallel components are needed to support power rating, one spare component is added to achieve $(n+1)$ redundancy. In this case the nominal and rated power of the parallel components are different and described by equation (1).

$P_{\text {rated }}=P / n$ and $P_{\text {nominal }}=P /(n+1)$

where, $\mathrm{P}$ is the power rating of the common bus bar.

Since the grid source is quite close to the drive beam, maximum fault currents are foreseen to be higher than the breaking currents of standard equipment. In order to increase impedance, and reduce the fault current at MV level, two HV/MV transformers in normal operation are never operated in parallel. Thus, there is only one $\mathrm{HV} / \mathrm{MV}$ transformer per MV bus bar and the redundancy must be provided from the MV side, by connecting the bus bars. In the case of transformer failure the power will be supplied from two neighboring bus bars, and all HV/MV transformers are rated at $3 / 2$ of the nominal power.

In the case of the failure of one cable, one transformer or one converter per bus bar, the system can still be operated with some reconfiguration. However, in the case of a bus bar failure, all loads corresponding to that bus bar will not be supplied. The bus bar failure rate is much lower than those of transformers, switchgears, cables and converters. However, two approaches are possible: first one, with radial distribution of bus bars, already explained; and the second with parallel bus bars. The illustration of single and double bus bar principle in case of MV bus bars is presented in Figure 3. In the second case, all loads and feeders associated with one bus bar have the potential to be connected to a parallel bus bar. Also, parallel bus bars can be connected amongst themselves as a support when there is a lack of power on one of them. For the case of double bus-bars, transformers are rated at twice the nominal power. The DC bus bar will remain single (without parallel pair) due to the large number of connections $(\sim 1300)$ providing that converters are rated according to equation (1).

Considering the typical transformers ratings and the equations for transformers nominal and rated powers, the scope for possible transformers number is calculated. The numbers of substations, HV/MV transformers (MV bus bars), $\mathrm{MV} / \mathrm{LV}$ transformers, LV bus bars, AC/DC converters and DC voltage bus bars are optimization variables, and overall system performance is dependent on these parameters.

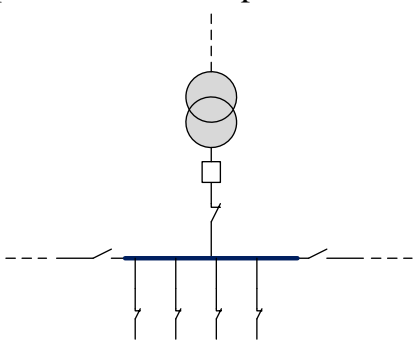

a)

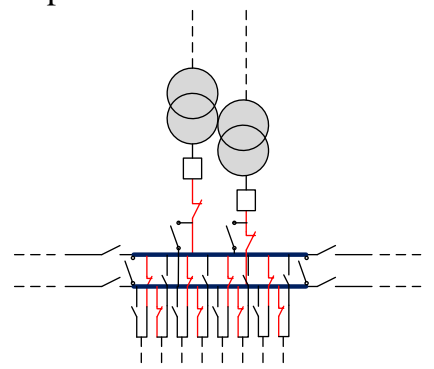

b)
Figure 3. Comparison of a) single and b) parallel bus bars design principle.

\section{CHARACTERISING VARIABLES FOR OPTIMISATION}

The selected grid layout described with the numbers of characteristic components, distribution voltages and design rules (single or double bus bar) should be evaluated. The evaluation includes efficiency and maximum fault current estimation, which need to be within the absolute constraints. Also, cost and unavailability must be estimated for comparison purposes, as a quality measurement between solutions.

For the purpose of efficiency and fault currents estimation, impedances of cables and transformers must be taken into account. Overhead lines are specific aluminum conductors, while all the other cables are halogen free XLPE copper conductors. Due to the limited spaces in switchgear cable sockets and loading issues of high cross section conductors, the maximum cross section used is $400 \mathrm{~mm}^{2}$. In the case of higher current ratings than those of $400 \mathrm{~mm}^{2}$ conductor, cable paralleling is applied. Based on current rating and voltage level, one or many cables are selected and impedance per unit length is extracted from the chosen cable catalogue. To have impedance estimation the cable length must be calculated.

Typical transformer impedances for specified power and primary voltage rating can be found in the catalogues, such as the ABB switchgear manual [8]. Per unit impedance $u_{k}$ and per unit resistance $u_{r}$ ranges taken for the manual are used for estimation of resistive and reactive part of the transformer impedance for specific voltage and power rating.

Cost models of the power system have not been obtained yet. They should include the prices of all power system components and all related civil engineering. Civil engineering involves buildings for the indoor substations and converters, concrete slabs for oil transformers, technical galleries for cable placement, etc. For the same number of power system components, the parallel bus bar solution is expected to have higher cost due to the larger overrating and higher quantities of switchgear. In that case, the higher availability is expected meaning that the design is a tradeoff between cost and availability. Efficiency and the maximum fault current should not be affected significantly by the bus bar layout. 


\subsection{EFFICIENCY}

The overall efficiency is estimated by taking into account losses in the entire system. Since the bus bars (and switchgear) have low impedance, they are assumed to be lossless. Cable losses are estimated by considering cable resistance, length and nominal current. For the same amount of power, higher voltage corresponds to lower currents and lower losses, justifying the distribution rule - the longest lines at the highest voltages. Transformer efficiency is estimated from the catalogue data of the transformers already used at CERN. Transformer losses include the no load loss (relating to the core loss), and full load loss relating to the winding loss (described by transformer winding resistance).

Converter losses depend on the topology and converter ratings. In the case of a Modular Multilevel Converter [9], containing internal storage elements, such as cell capacitors and arm inductors the losses relate to both semiconductor losses and losses in passive elements. In the case of lower power and voltage levels, simplest topologies such as Neutral Point Clamped [10] only switching and conduction losses of semiconductors are included. In all cases, semiconductor losses are estimated using the waveform averaging method proposed in [11]. Together with the analytical approach the results are verified using PLECs simulation environment.

\subsection{FAULT CURRENTS}

The maximum fault current in the system should not exceed 20kA which is the breaking current of standard MV interruption devices. Usage of standard equipment lowers the price and also improves the overall availability of the system.

Typically, 3 phase to ground faults are considered the most severe. For this, symmetrical type of fault, only positive sequence currents and impedances need to be calculated. In cases of some asymmetrical faults, e. g. two phases to ground, zero and negative (equal to positive sequence) sequence impedance need to be obtained. Considering the current CERN network, HV/MV transformers are YnYnd0, so that the zero sequence current can pass from primary to secondary, through to the zero sequence transformer impedances and neutral grounding impedances. Thus, the zero sequence current exists in case of some asymmetrical faults on the MV level. Current MV/LV transformers are Ynd11, blocking zero sequence fault current on the LV connection [12].

The fault currents are calculated by using equivalent circuit containing impedances towards the grid and an equivalent voltage source at the fault location. The equivalent voltage source $U_{\text {fault }}$ as a function of nominal voltage $U_{\text {nom }}$ at the fault location is given with the equation (2).

$U_{\text {fault }}=c_{\text {max }} \cdot U_{\text {nom }} / \sqrt{3} \approx 1.1 \cdot U_{\text {nom }} / \sqrt{3}$

where, $c_{\max }$ is the voltage factor for calculating fault current maximum.

The grid maximum fault power and impedance ratios given in the Table 1 are used for estimating the minimum grid resistance and reactance in case of positive and zero sequence. Similarly, overhead lines and cable impedances are estimated.
Transformer per unit impedance ranges are used for the positive and zero sequence impedance estimations. All per unit impedances are transferred to the absolute values with the base voltage equal to the nominal voltage at the fault location.

\subsection{AVAILABILITY}

Availability of the power systems considered, need to be evaluated for the purpose of solution comparison. In both cases of single and parallel radially distributed bus bars, a certain amount of redundancy is applied, so in the case of failure of one element, the network can be reconfigured to supply the loads. In some cases, the system should be powered off, reconfigured and restarted again. However, since this kind of failure is possible to solve by reconfiguration, it is not considered as a fault. Only the failures that can not be eliminated by reconfiguration are considered as faults.

Fault tree analysis [13] is applied for availability estimation. The analysis is based on the most undesired event, referred to as a top event. In the case of the CLIC, the top event would be the situation in which one or more klystron modulators are not supplied with the appropriate DC voltage. However, since the failure on any of the voltage levels can cause the top event, the power system is divided into sectors. Every sector has its internal top event that directly causes a certain amount of power not to be delivered to the klystrons modulators.

Fault tree analysis is based on the collection of all combinations of basic events that are leading to the top event [13]. Failure of any component is considered to be the basic event (BE). Combination of basic events that lead a top event is called a minimum cut set (MCS). Any of the MCSs can cause the top event, which is in that case described by equation (3).

$\left.T E=\sum_{i} M S C_{i}=\sum_{i}\left(\prod_{j} B E\right)_{j}\right)_{i}$

The probability of the top event is calculated with respect of Boolean algebra rules, taking into account that some of MSC are not mutually exclusive.

In the case of single radially distributed bus bars, the top event on one voltage level relates to the lack of power feeding one of the bus bars or failure on the bus bar itself. In the case of double bus bar, the top event at one voltage level relate to the lack of total amount of power delivered to both parallel bus bars, failure of both parallel bus bars, or failure on one without ability to redirect all the loads to the parallel bus bar.

For the probability (to fail) calculation, the data for the all basic events probabilities must be known. This data is usually statistical based on experience of the cables, switchgear, circuit breakers, transformers, and switch failure rates. The data is obtained from the German Association for Electrical, Electronic \& Information Technologies (VDE) that collected the failure rates for the transmission and distribution network components [14]. Obtained data relates to the period from 2004 to 2011 and it is described with the number of failed components, failure frequency [1/year], average interruption duration $[\mathrm{h}]$ and probability of component unavailability 
[min/year].

Applying this method, the typical unavailability of parallel and single bus bar system for the same number of components is $20 \mathrm{~min} /$ year and $170 \mathrm{~min} /$ year, respectively. Since most of the network reconfigurations involve system turn off, realistically, the difference in the availability is lower.

\section{POWER ELECTRONICS}

Once the optimal layout is found the power converter interface can be defined. Various AC/DC converter topologies were considered, but by considering of DC voltage level, topological selections are made. In the case of DC voltages above $10 \mathrm{kV}$ DC and powers above $2 \mathrm{MW}$ modular multilevel topologies are selected. Apart from high efficiency, topologies such as MMC and Parallel Hybrid Modular Multilevel Converter (PH-M2C) introduced in [5] offer a good AC waveform quality and high redundancy, due to the cell structure of MMC chain links. However, in the case of lower voltages and powers, the lack of power density of an MMC becomes significant and they are not considered cost-effective when compared to the simplest topologies such as the 3-level NPC converter. The NPC converter has higher semiconductor losses and lower AC waveforms quality, due to the higher switching frequency and three-level operation.

Considering the bus bars structure and paralleling of the converters, NPC converter AC waveforms can be improved by the canceling switching harmonics with phase shifted modulation among the converters. From the DC side, parallel connection of MMC or NPC converters is seen as one large converter with many phase legs attached to three phases only. This does not complicate control algorithm, when compared to single converter case. However in the case of MPC, paralleling of the converter outputs might cause issues with control.

\subsection{CONTROL}

During the pulse, the modulator discharges the capacitor providing the DC voltage to droop. The main objective of the converter control is to maintain low power fluctuation on the AC side regardless the $50 \mathrm{~Hz}$ power fluctuation and capacitor discharge on the DC side; and maximizing DC voltage repeatability. The collaboration team is working on reducing the power fluctuation on the DC side, by using a fast voltage compensator principle [7]. The team is working on the pulse quality and flat top repeatability, using the methods that rely on a repeatable DC voltage at the beginning of the pulse.

The traditional current control based algorithms do not react to rapid variation of DC voltage caused by the klystron modulator pulse discharge, meaning that those changes do not propagate to the generated references. Measured DC voltage waveform will reflect the discharge, and once it is fed to modulation block, it will correct the gate drive signals and the AC voltage, "hiding" the $50 \mathrm{~Hz}$ harmonics from the AC side. However, the modular multilevel converters that contain internal storage elements need some control modification to maintain the energy stored in converter. The control approach is decoupled AC and DC side control, proposed in [15]. The AC power is controlled by the $d, q$ current control providing the AC part of the arm voltage reference. The DC side control relies on the control of stored energy in each of the phases, providing the circulating phase current reference. Fast current control produces the DC part of the arm voltage reference.

The described algorithm is capable of suppressing the AC power fluctuation below the limiting $2 \%$. However, the algorithm is not controlling the DC voltage. For the purpose of DC voltage repeatability control, repetitive control algorithms are considered. Repetitive controllers are not aimed of the system stabilization, but to correct the periodical errors in the system. Repetitive controllers work together with the traditional regulator for the DC bus, providing better performance. The idea is to collect the signal history from previous pulses in order to act in the next modulator discharge pulse and correct the pre-pulse charging voltage.

\section{RESULTS}

A power system with single radially distributed bus bars, applying $(n+1)$ redundancy, on all voltage levels, except MV where the redundancy is achieved by bus bar connections, is analyzed. At first, the DC voltage is set to $10 \mathrm{kV}$ (LV to $6 \mathrm{kV}$ and $\mathrm{MV}$ to $30 \mathrm{kV}$ ). The MMC approaches are considered for the power electronics. All possible solutions are generated by the substations number from 1 to 4 , HV/MV transformers number from 3 to 7, number of LV bus bars from 10 to 14, etc. The fault currents are observed for the population of all solutions, and presented in Figure 4. The 20 kA line presents the breaking current limitation, showing that only solutions of 6 and 7 HV/MV transformers provide satisfactory fault currents.

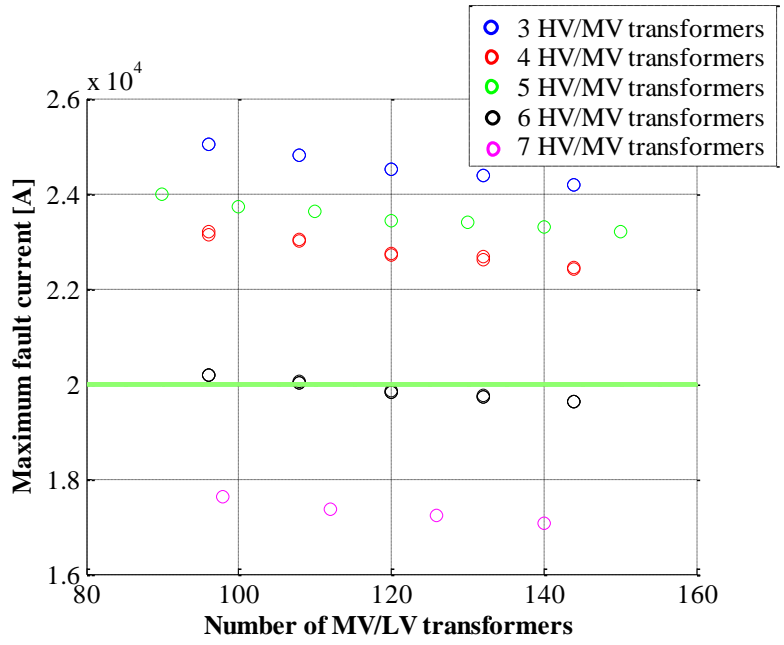

Figure 4. Estimated fault current maximum for the all solutions corresponding to $10 \mathrm{kV}$ DC voltage.

The efficiency evaluation of all solutions satisfying the fault current constraint is presented in Figure 5. The highest efficiency of $97.73 \%$ is achieved with 3 substations, $6 \mathrm{HV} / \mathrm{MV}$ transformers, $12 \mathrm{LV}$ bus bars, $120 \mathrm{MV} / \mathrm{LV}$ transformers and 144 converters. All feasible solutions have an overall 
efficiency higher than $97.4 \%$. The efficiency increases with increased number of converters. In that case power density and cost effectiveness is lower. The unavailability of all feasible solutions is between 167 and 174 min/year. Better availability can be achieved with parallel bus bar principle but this will increase the cost.

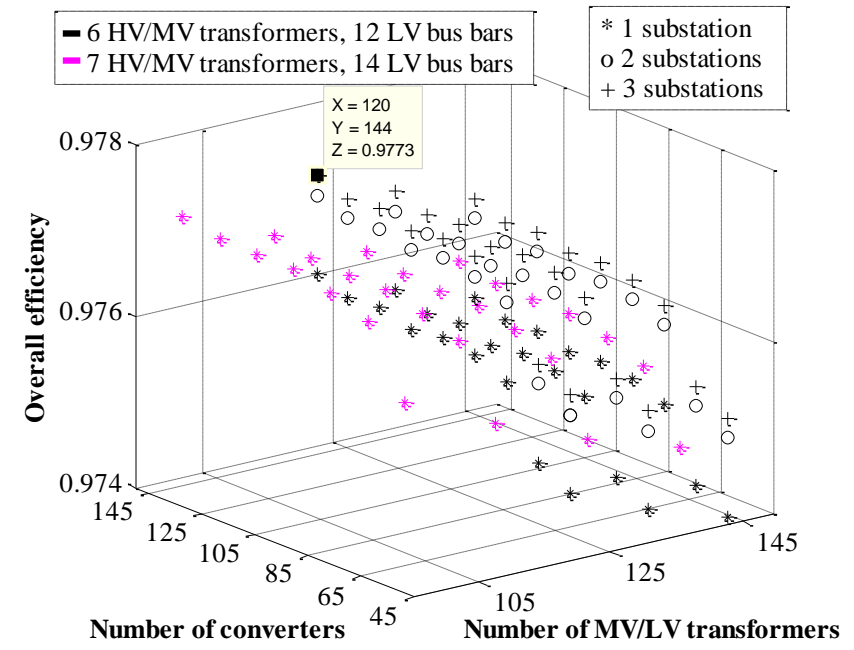

Figure 5. Estimated efficiency of all feasible solutions corresponding to $10 \mathrm{kV}$ DC voltage, as a function of number of transformers and converters.

If the DC voltage is fixed to $20 \mathrm{kV}$, low AC voltage should be $11 \mathrm{kV}$ and medium voltage $30 \mathrm{kV}$. The MMC topology is used, but with a larger number of cells per arm/phase, when compared with $10 \mathrm{kV}$ DC voltage case. Solutions with acceptable fault currents are only in the case of 6 and 7 $\mathrm{HV} / \mathrm{MV}$ transformers. The best efficiency is $97.8 \%$ (3 substations, $6 \mathrm{HV} / \mathrm{MV}$ transformers, $6 \mathrm{LV}$ bus bars, $90 \mathrm{MV} / \mathrm{LV}$ transformers and 72 converters) while all solutions have efficiency higher than $97.3 \%$. Unavailability of all solutions is between 166 and $175 \mathrm{~min} /$ year.

The lowest $\mathrm{AC}$ voltage considered is $3.3 \mathrm{kV}$, which in case of NPC converters provides $6 \mathrm{kV}$ DC output voltage. In this case due to the low AC voltage level, the maximum fault currents are above $25 \mathrm{kA}$. Additionally, for $2.5 \mathrm{kHz}$ switching frequency converter efficiency is approximately $97.2 \%$ providing the overall system efficiency of around $96 \%$. Thus, when the DC voltage is below $10 \mathrm{kV}, \mathrm{MV} / \mathrm{LV}$ transformers impedances need to increase to lower the fault currents, but there is no scope for increasing the losses, due to higher NPC converter losses.

\section{CONCLUSION}

This paper presented a progress on finding the optimal grid layout solution of CLIC klystron modulators. The optimization methodology is presented, involving the importance of the parameters such as efficiency, fault currents, unavailability and cost. The absolute limitations of the grid source at CERN are also respected. The power electronics considerations including topology selection, and control aspects are presented.

The optimization results without cost functions are shown. Overall efficiencies above $97 \%$ are achieved for all solutions using MMC converters (DC voltage above $10 \mathrm{kV}$ ). The efficiency increases with the increase of number of converters (with fixed number of other components), i.e. decrease of power density. Assuming that the efficiency requirement is met, the cost parameter might move the optimal solution towards lower number of converters. Unavailability of all single radially distributed bus bars is similar - between 165 and $175 \mathrm{~min} /$ year. The higher availability can be provided by using parallel bus bars, with an increase of cost, and similar values of fault currents and efficiency.

The next stage of optimization will include financial aspects of solution and weighted cost function as a tradeoff of evaluated parameters. Once the optimal converter ratings are set, the topology can be selected. The repetitive controllers will be implemented in parallel to power control to meet low $\mathrm{AC}$ power fluctuation and repetitive DC voltage requirements.

\section{REFERENCES}

[1] D. Dannheim, P. Lebrun, L. Linssen, D. Schulte, and S. Stapnes. CLIC e+e- Linear Collider Studies - Input to the Snowmass process 2013 [Online].

[2] D. Aguglia, C. A. Martins, M. C. Bastos, D. Nisbet, D. Siemaszko, E. Sklavounou, and P. Viarouge, "Klystron modulator technology challenges for the Compact Linear Collider (CLIC)," in Pulsed Power Conference (PPC), 2011 IEEE, 2011, pp. 1413-1421.

[3] K. D. Papastergiou and D. Aguglia, "Power System design compromises for large-scale linear particle accelerators," in Power Electronics and Applications (EPE), 2013 15th European Conference on, 2013, pp. 1-9.

[4] R. Marquardt, "Modular Multilevel Converter: An universal concept for HVDC-Networks and extended DC-Bus-applications," in Power Electronics Conference (IPEC), 2010 International, 2010, pp. 502-507.

[5] R. Feldman, M. Tomasini, J. C. Clare, P. Wheeler, D. R. Trainer, and R. S. Whitehouse, "A low loss modular multilevel voltage source converter for HVDC power transmission and reactive power compensation," in $A C$ and DC Power Transmission, 2010. ACDC. 9th IET International Conference on, 2010, pp. 1-5.

[6] M. Jankovic, A. Watson, J. Clare, P. Wheeler, and D. Aguglia, "Grid interface challenges and candidate solutions for the Compact Linear Collider's (CLIC) klystron modulators," in Pulsed Power Conference (PPC), 2013 19th IEEE, 2013, pp. 1-6.

[7] F. C. Magallanes, D. Aguglia, P. Viarouge, and J. Cros, "Design of a solid-state fast voltage compensator for klystron modulators requiring constant AC power consumption," in IPMHVC 2014, 2014 IEEE, 2014, pp. 1-4.

[8] (2012, 27/05/2014). ABB Switchgear Manual (12 ed.) [User manual].

[9] A. Lesnicar and R. Marquardt, "An innovative modular multilevel converter topology suitable for a wide power range," in Power Tech Conference Proceedings, 2003 IEEE Bologna, 2003, p. 6 pp. Vol.3.

[10] J. Rodriguez, L. Jih-Sheng, and P. Fang Zheng, "Multilevel inverters: a survey of topologies, controls, and applications," Industrial Electronics, IEEE Transactions on, vol. 49, pp. 724-738, 2002.

[11] S. Allebrod, R. Hamerski, and R. Marquardt, "New transformerless, scalable Modular Multilevel Converters for HVDC-transmission," in Power Electronics Specialists Conference, 2008. PESC 2008. IEEE, 2008, pp. 174-179.

[12] Y. Hase, Handbook of Power System Engineering: Wiley, 2007.

[13] M. Čepin, Assessment of Power System Reliability: Methods and Applications: Springer London, 2011.

[14] H. Vennegeerts. Ermittlung von Eingangsdaten zur Zuverlässigkeitsberechnung aus der FNN-Störungsstatistik - Neue Auswertung der Berichtsjahre 2004 - 2011 [Online].

[15] M. Hagiwara and H. Akagi, "Control and Experiment of PulsewidthModulated Modular Multilevel Converters," Power Electronics, IEEE Transactions on, vol. 24, pp. 1737-1746, 2009. 